\title{
Crystal Structure of 1,3-Bis(3,5-dibromosalicylideneamino)-2-propanol
}

\author{
Masahiro Mikuriya, ${ }^{* \dagger}$ Nagisa Tsuchimoto, ${ }^{*}$ Yoshiki Koyama, ${ }^{*}$ Ryoji Mitsuhashi, ${ }^{* *}$ and \\ Motohiro TsUBOI* \\ *Department of Applied Chemistry for Environment and Research Center for Coordination Molecule-based \\ Devices, School of Biological and Environmental Sciences, Kwansei Gakuin University, 2-1 Gakuen, \\ Sanda 669-1337, Japan \\ **Institute of Liberal Arts and Science, Kanazawa University, Kakuma, Kanazawa 920-1192, Japan
}

\begin{abstract}
The crystal structure of 1,3-bis(3,5-dibromosalicylideneamino)-2-propanol was determined by the single-crystal X-ray diffraction method at $90 \mathrm{~K}$. It crystallizes in the monoclinic space group $P 2_{1} / n$ with $a=16.5374(17) \AA, b=18.1164(19) \AA$, $c=19.330(2) \AA, \beta=93.608(3)^{\circ}, V=5779.9(10) \AA^{3}, D_{\mathrm{x}}=2.117 \mathrm{~g} / \mathrm{cm}^{3}$, and $Z=12$. The $R 1[I>2 \sigma(I)]$ and $w R 2$ (all data) values are 0.0743 and 0.1541 , respectively, for all 13203 independent reflections. The crystal contains three crystallographically independent Schiff-base molecules. Each molecule takes a bended structure at the central carbon atom with a dihedral angle of between the two 3,5-dibromosalicylideneaminomethyl planes of $79.68,84.52$, or $89.72^{\circ}$. The three Schiff-base molecules are connected one another by intermolecular hydrogen bonds between an alcohol-oxygen atom and a phenolato-oxygen atom of a neighboring Schiff-base molecule.
\end{abstract}

(Received August 7, 2021; Accepted August 20, 2021; Published on web January 10, 2022)

Schiff-base molecules, 1,3-bis(salicylideneamino)-2-propanol $\left(\mathrm{H}_{3} \mathrm{salpr}\right)$, and their substituted derivatives, are potential pentadentate ligands with two phenolato-oxygen, two iminonitrogen, and one central alcoholate-oxygen donor atoms by deprotonation with coordination to metal atoms. It is known that these Schiff-base ligands form a mononuclear copper(II) complex as a tetradentate $O, N, N, O$-chelate, ${ }^{1}$ and dinuclear copper(II), ${ }^{2}$ nickel(II), ${ }^{3}$ manganese(III),${ }^{4}$ and $\operatorname{cobalt(III)}{ }^{5}$ complexes as tridentate $O, N, O$-chelates. Previously, we reported on the crystal structures of a monosubstituted Schiffbase ligand, 1,3-bis(5-nitrosalicylideneamino)-2-propanol $\left(\mathrm{H}_{3} \text { nsalpr }\right)^{6}$ and a disubstituted Schiff-base ligand, 1,3-bis $(5-$ chloro-3-methoxysalicylideneamino)-2-propanol ( $\left.\mathrm{H}_{3} \mathrm{cmsalpr}\right){ }^{7}$ The former has one nitro group at the salicylideneamino ring, whereas the latter has one chloro group and one methoxy group at the salicylideneamino ring. In this study, we prepared 1,3-bis(3,5-dibromosalicylideneamino)-2-propanol ( $\left.\mathrm{H}_{3} \mathrm{dbsalpr}\right)$, which has two bromo groups at the salicylideneamino ring (Fig. 1), and determined the crystal structure of $\mathrm{H}_{3} \mathrm{dbsalpr}$,

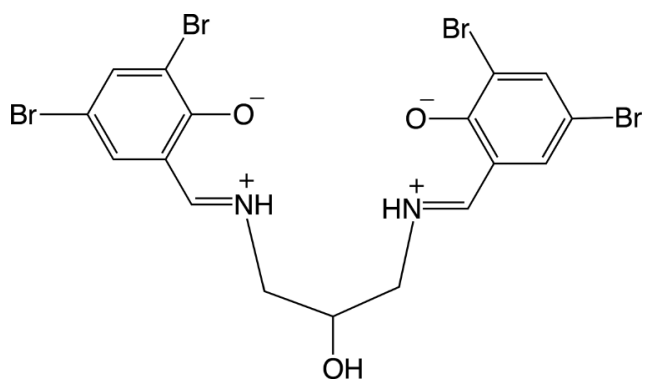

Fig. 1 Chemical structure.

$\dagger$ To whom correspondence should be addressed.

E-mail: junpei@kwansei.ac.jp which has not been elucidated so far.

1,3-Diamino-2-propanol (0.646 g, $7 \mathrm{mmol})$ and 3,5-dibromosalicylaldehyde $(4.00 \mathrm{~g}, 14 \mathrm{mmol})$ were dissolved in methanol $\left(50 \mathrm{~cm}^{3}\right)$-acetonitrile $\left(10 \mathrm{~cm}^{3}\right)$. The solution was refluxed for $3 \mathrm{~h}$, and then left at room temperature. The resulting yellow precipitate was filtered off, washed with methanol, and dried under a vacuum. Yield, $3.98 \mathrm{~g} \mathrm{(91 \% ).} \mathrm{IR}\left(\mathrm{KBr}, \mathrm{cm}^{-1}\right): 3235$ $(v(\mathrm{OH})), 3055\left(v(\mathrm{CH}(\right.$ phenyl $)), 2925\left(v_{\mathrm{as}} \mathrm{CH}_{2}\right), 2889\left(v_{\mathrm{s}} \mathrm{CH}_{2}\right)$, $1632(v \mathrm{C}=\mathrm{N})$. X-ray quality crystals were grown by recrystallization from acetonitrile.

X-ray diffraction data were collected on a Bruker CCD X-ray diffractometer (SMART APEX) using graphite-monochromated Mo- $K \alpha$ radiation. Crystal data and details concerning the data collection are given in Table 1 . The structure was solved by an intrinsic phasing method with SHELXT-2014, and refined by full-matrix least-squares methods with SHELXL-2018. The hydrogen atoms were inserted at their calculated positions, and fixed there with restraints. The hydrogen atoms at the imino groups were also inserted at the calculated positions because it was difficult to locate the hydrogen atoms from the D-Fourier maps including the phenolate oxygen areas. For thermal anisotropic displacement parameters (ADPs) of most of the carbon atoms, enhanced rigid bond restraints were applied. Crystallographic data have been deposited with Cambridge Crystallographic Data Centre (Deposit number CCDC2091176). Copies of the data can be obtained free of charge via http://www.ccdc.cam.ac.uk/conts/retrieving.html (or from the Cambridge Crystallographic Data Centre, 12, Union Road, Cambridge, CB2 1EZ, UK; Fax, +44 1223 336033; e-mail, deposit@ccdc.cam.ac.uk).

In the crystal, the asymmetric unit consists of three independent 1,3-bis(3,5-dibromosalicylideneamino)-2-propanol, $\mathrm{H}_{3} \mathrm{dbsalpr}$ molecules, like in the case for 1,3-bis(salicylideneamino)-2propanol (four independent molecules in the asymmetric unit). ${ }^{8}$ The asymmetric unit drawn with the thermal ellipsoids is shown in Fig. 2. Selected bond distances and angles are given in 
Table 1 Crystal and experimental data

Chemical formula: $\mathrm{C}_{17} \mathrm{H}_{14} \mathrm{Br}_{4} \mathrm{~N}_{2} \mathrm{O}_{3}$
Formula weight $=613.94$
$T=90 \mathrm{~K}$
Crystal system: monoclinic
Space group: $P 2_{1} / n$
$a=16.5374(17) \AA$
$b=18.1164(19) \AA$
$c=19.330(2) \AA$
$V=5779.9(10) \AA^{3}$
$Z=12$
$D_{\mathrm{x}}=2.117 \mathrm{~g} / \mathrm{cm}^{3}$
Radiation: Mo $K \alpha(\lambda=0.71073 \AA)$
$\mu($ Mo $K \alpha)=8.375$ mm

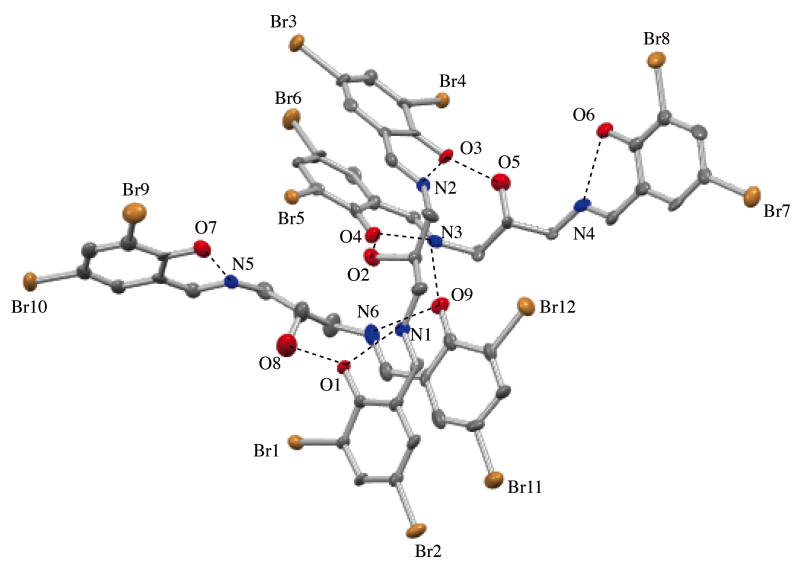

Fig. 2 Asymmetric unit of the crystal structure of the title compound, showing hydrogen bonds as dashed lines. The thermal ellipsoids are shown at the 50\% probability level. Hydrogen atoms are omitted for clarity.

Table 2. The N1-C7, N2-C11, N3-C24, N4-C28, N5-C41, and N6-C45 distances of the three $\mathrm{H}_{3}$ dbsalpr molecules range from 1.257(12) to 1.302(12) $\mathrm{A}$, which are normal as $\mathrm{C}=\mathrm{N}$ double-bond (average 1.281(13) $\mathrm{A}),{ }^{9}$ whereas the N1-C8, N2-C10, N3-C25, $\mathrm{N} 4-\mathrm{C} 27, \mathrm{~N} 5-\mathrm{C} 42$, and $\mathrm{N} 6-\mathrm{C} 44$ distances are in the range of $1.444(13)-1.453(12) \AA$, which can be considered to be of the C-N single-bond character (average 1.469(14) $\mathrm{A}) .{ }^{9} \quad$ The bond angles around the imino-nitrogen atom (C7-N1-C8, C10-N2C11, C24-N3-C25, C27-N4-C28, C41-N5-C42, and C44-N6$\mathrm{C} 45)$ are $122.4(9)-127.4(10)^{\circ}$, supporting the presence of a bonded hydrogen atom. ${ }^{6,10}$ The O1-C1, O3-C17, O4-C18,
Table 2 Selected bond distances $(\AA)$ and angles $\left({ }^{\circ}\right)$

\begin{tabular}{llll}
\hline O1-C1 & $1.278(11)$ & N4-C28 & $1.257(12)$ \\
O2-C9 & $1.416(11)$ & Br5-C19 & $1.864(10)$ \\
O3-C17 & $1.273(11)$ & Br6-C21 & $1.916(9)$ \\
N1-C7 & $1.288(11)$ & Br7-C31 & $1.898(11)$ \\
N1-C8 & $1.460(12)$ & Br8-C33 & $1.873(11)$ \\
N2-C10 & $1.486(12)$ & O7-C35 & $1.267(12)$ \\
N2-C11 & $1.302(12)$ & O8-C43 & $1.348(13)$ \\
Br1-C2 & $1.870(10)$ & O9-C51 & $1.232(12)$ \\
Br2-C4 & $1.877(10)$ & N5-C41 & $1.280(12)$ \\
Br3-C14 & $1.902(10)$ & N5-C42 & $1.449(13)$ \\
Br4-C16 & $1.877(10)$ & N6-C44 & $1.444(13)$ \\
O5-C26 & $1.404(11)$ & N6-C45 & $1.295(13)$ \\
O4-C18 & $1.262(12)$ & Br9-C36 & $1.902(12)$ \\
O6-C34 & $1.324(11)$ & Br10-C38 & $1.878(11)$ \\
N3-C24 & $1.283(12)$ & Br11-C48 & $1.904(10)$ \\
N3-C25 & $1.449(13)$ & Br12-C50 & $1.909(10)$ \\
N4-C27 & $1.453(12)$ & & \\
C7-N1-C8 & $126.7(9)$ & C27-N4-C28 & $122.4(9)$ \\
C10-N2-C11 & $122.9(9)$ & C41-N5-C42 & $123.6(9)$ \\
C24-N3-C25 & $125.1(10)$ & C44-N6-C45 & $127.4(10)$ \\
\hline
\end{tabular}

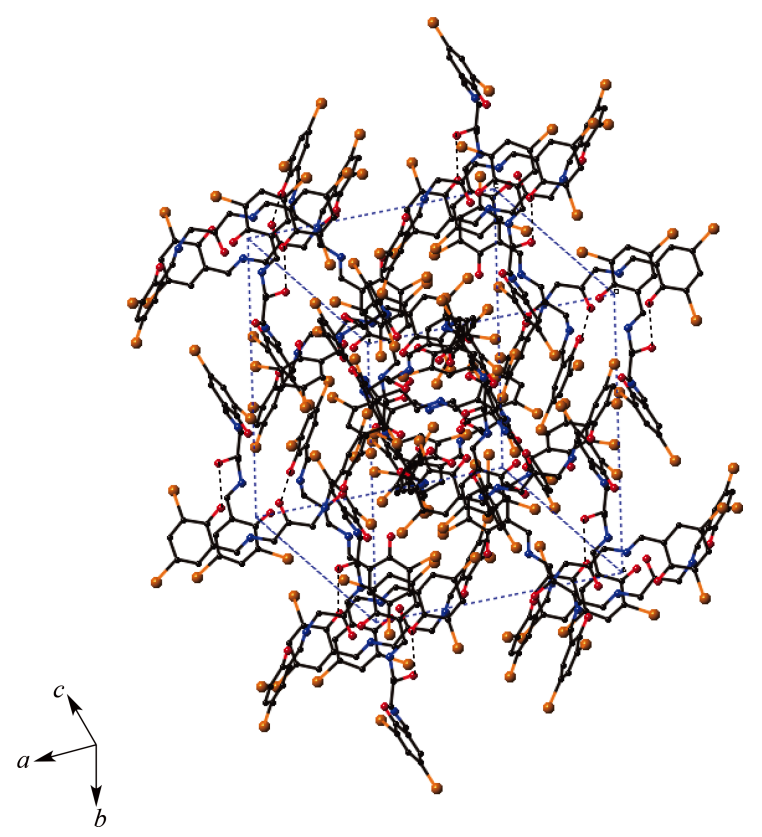

Fig. 3 Packing diagram of the title compound, showing hydrogen bonds (dashed lines).

O6-C34, O7-C35, and O9-C51 (1.232(12) - 1.324(11) ̊) bond lengths are a little shorter than the usual $\mathrm{C}$ (aromatic)-O bond lengths (average 1.362(15) $\mathrm{A}) .{ }^{9} \quad$ The $\mathrm{C}-\mathrm{O}$ shortening and the variation of the $\mathrm{C}-\mathrm{C}$ distances of the phenolate aromatic rings suggest some contribution of a quinoid-like structure for the phenolate rings. This structural feature was found in the related Schiff-base ligands, 1,3-bis(5-nitrosalicylideneamino)-2-propanol $\left(\mathrm{H}_{3} \mathrm{nsalpr}\right)^{6}$ and 1,3-bis(5-chloro-3-methoxysalicylideneamino)2-propanol $\quad\left(\mathrm{H}_{3} \mathrm{cmsalpr}\right){ }^{7} \quad \mathrm{~N}$-salicylidene-2-hydroxy-5-chlorobenzylamine, ${ }^{11}$ and $\quad \mathrm{N}$-salicylidene-2-hydroxy-5-bromobenzylamine. ${ }^{12}$ The $\mathrm{Br}-\mathrm{C}$ distances range from 1.864(10) to

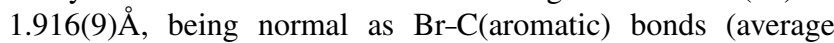
$1.899(12) \AA)^{9}{ }^{9}$ The three Schiff-base molecules have a similar bended structure, where the two approximate planes of the 3,5-dibromosalicylideneaminomethyl $\mathrm{C}_{8} \mathrm{Br}_{2} \mathrm{NO}$ moieties in each 
ligand are not coplanar, with a dihedral angle of $79.68,84.52$, or $89.72^{\circ}$ at the central $\mathrm{C}$ atom, like the case for disubstituted 1,3-bis(salicylideneamino)-2-propanol, $\mathrm{H}_{3} \mathrm{cmsalpr} \quad\left(74.71^{\circ}\right) .^{7}$ This is in contrast with the case for monosubstituted 1,3-bis(5nitrosalicylideneamino)-2-propanol, $\mathrm{H}_{3}$ nsalpr $\left(11.21^{\circ}\right) .^{6}$ In each Schiff-base molecule, there are intramolecular hydrogen bonds between imino-nitrogen and phenolato-oxygen atoms [N1-

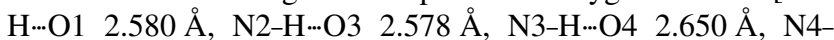

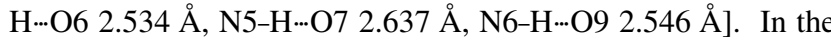
crystal, the three Schiff-base molecules are connected one another by intermolecular hydrogen bonds between the alcoholoxygen atom and the phenolato-oxygen atom of neighboring Schiff-base molecule [O2-H…04 2.826 ̊, O5-H…03 $2.770 \AA$, O8-H...O1 2.725 $\AA$ ] (Figs. 2 and 3), similar to the case for $\mathrm{H}_{2}$ salpr. $^{10}$

\section{Acknowledgements}

The present work was partially supported by Grants-in-Aid for Scientific Research No. 17K05820 from the Ministry of Education, Culture, Sports, Science and Technology and the MEXT-Supported Program for the Strategic Research Foundation at Private Universities, 2010 - 2014.

\section{References}

1. E. Donmez, H. Kara, A. Karakaş, H. Ünver, and A. Elmali,
Spectrochim. Acta, 2007, A66, 1141.

2. Y. Nishida and S. Kida, J. Chem. Soc., Dalton Trans., 1986, 2633.

3. M. Mikuriya, T. Sasaki, A. Anjiki, S. Ikenoue, and T. Tokii, Bull. Chem. Soc. Jpn., 1992, 65, 334.

4. M. Mikuriya, Y. Yamato, and T. Tokii, Bull. Chem. Soc. Jpn., 1992, 65, 1466.

5. M. Mikuriya, Y. Naka, D. Yoshioka, and M. Handa, X-ray Struct. Anal. Online, 2016, 32, 55.

6. M. Mikuriya, Y. Naka, and D. Yoshioka, X-ray Struct. Anal. Online, 2015, 31, 55. Erratum: X-ray Struct. Anal. Online, 2016, 32, 11.

7. M. Mikuriya, Y. Koyama, and R. Mitsuhashi, X-ray Struct. Anal. Online, 2019, 35, 33.

8. M. Azam, Z. Hussain, I. Warad, S. I. Al-Resayes, M. S. Khan, M. Shakir, A. Trzesowska-Kruszynska, and R. Kruszyaski, Dalton Trans., 2012, 41, 10854.

9. S. Ohba and H. Uekusa, "Introduction to X-ray Crystal Structure Analysis" (Japanese), 2014, Kagakudojin, Kyoto.

10. M. T. Kaczmarek, M. Skrobanska, M. Zabiszak, M. WalesaChorab, M. Kubicki, and R. Jastrzab, RSC Adv., 2018, 8, 30994.

11. Y. Kakuta, C. Myojo, D. Yoshioka, Z. Zhu, and M. Mikuriya, Anal. Sci., 2008, 24, x267.

12. N. Masuda, Y. Kakuta, D. Yoshioka, and M. Mikuriya, $X$-ray Struct. Anal. Online, 2014, 30, 7. 